\title{
Evaluación del Sistema de Modelamiento Hidrológico HEC- HMS para la Simulación Hidrológica de una Microcuenca Andina Tropical
}

\author{
Paola Duque-Sarango*, Daysi M. Patiño y Xavier E. López \\ Universidad Politécnica Salesiana-Ecuador, INBIAM Grupo de Investigación en Biotecnología Ambiental. Calle Vieja 12 - \\ 30 y Elia Liut, Casilla 46 sector 2, Cuenca-Ecuador. (e-mail: pduque@ups.edu.ec; dpatinor1@est.ups.edu.ec, \\ xlopezu@est.ups.edu.ec).
}

* Autor a quien debe ser dirigida la correspondencia

Recibido Mar. 5, 2019; Aceptado Abr. 30, 2019; Versión final May. 27, 2019, Publicado Dic. 2019

\begin{abstract}
Resumen
Se simuló el comportamiento hidrológico en una cuenca tropical en los Andes de Ecuador, conocida como la microcuenca del río Chaquilcay, en la provincia de Azuay, en Ecuador. Se obtienen hidrogramas para la determinación de caudales y para generar información hidrológica base para la gestión de cuencas, sobre todo en una zona donde no existen estudios de este tipo. Se utilizaron curvas intensidad-duración-frecuencia (IDF) para la construcción de hietogramas. Con esta información, se aplicó un sistema de modelamiento hidrológico con la herramienta HEC-HMS (versión 4.1), y el programa ArcMap 10.1 para determinar las características morfológicas de la cuenca. Se logró obtener un caudal punta en el desagüe de $1.7 \mathrm{~m} 3 / \mathrm{s}, 1.8$ $\mathrm{m}^{3} / \mathrm{s}$ y $1,9 \mathrm{~m} 3 / \mathrm{s}$ para períodos de retorno de 25, 50 y 100 años respectivamente. Esta investigación puede ser útil para cuencas hidrográficas en el área de la región ecuatoriana, que tienen una función importante en el ecosistema y que no cuentan con información hidrometeorológica.
\end{abstract}

\section{Evaluation of the Hydrological Modeling System HEC-HMS for the Hydrological Simulation of a Tropical Andean Micro-basin}

\begin{abstract}
The present study simulated the hydrological behavior in a tropical catchment in the Andes of Ecuador, known as the Chaquilcay micro basin, situated in the province of Azuay, in Ecuador. Hydrographs to determine flows are obtained and for generating hydrological information about micro basins, especially in zones where this type of studies do not exist. Intensity, duration-frequency curves (IDF) were used for the construction of hietograms. With this information, a hydrological modeling system was applied, with the HEC-HMS tool (version 4.1), and the ArcMap 10.1 program to determine the morphological characteristics of the basin. It was possible to obtain a peak flow in the drainage of $1.7 \mathrm{~m}^{3} / \mathrm{s}, 1.8 \mathrm{~m}^{3} / \mathrm{s} 1.9 \mathrm{~m}^{3} / \mathrm{s}$ for return periods of 25,50 and 100 years respectively. This investigation can be useful for hydrographic basins in the area of the Ecuadorian region, which have an important function in the ecosystem but do not have hydro meteorological information.
\end{abstract}

Keywords: flow modeling; HEC-HMS; hydrograph; water resource; tropical hydrology 


\section{INTRODUCCIÓN}

La importancia del recurso hídrico radica en la relación con los cambios climáticos, meteorológicos y los procesos de precipitación, evaporación que se presentan a corto y largo plazo (Gutiérrez 2014). Los cambios globales que implican cambios simultáneos y rápidos tanto en la temperatura de la superficie de la Tierra como en la cobertura del suelo están provocando cambios profundos en el balance global del agua, el ciclo del carbono y los ecosistemas de la Tierra (Aber et al., 2001). Los ecosistemas de alta montaña son particularmente vulnerables a los impactos de estos cambios globales, con cambios documentados en la distribución de las especies, la composición de la comunidad y las tasas de crecimiento de la vegetación (Dirnböck et al., 2003; Kulonen et al., 2017, Quintero et.al., 2017). Sin duda, estos cambios alterarán el balance del agua de captación, a través de la captación diferencial de agua y carbono por parte de las plantas a medida que cambian la distribución y la eficiencia del uso del agua (Acosta et. al., 2014, Cargua et.al., 2014.)

Por otro lado los Andes Tropicales son una de las regiones hidrológicamente más diversas del mundo, debido a la convergencia de los sistemas climáticos del Pacífico ecuatoriano y amazónico, combinados con un terreno excesivamente escarpado y un alto nivel de biodiversidad vegetal (Celleri et al., 2007; Padrón et al., 2015). Las praderas alpinas tropicales de los Andes del norte, comúnmente conocidas como páramo, proporcionan abundante agua de alta calidad para las poblaciones río abajo, así como una variedad de otros servicios ambientales (Mosquera et. al., 2015). Este ecosistema se encuentra en los Andes superiores de Venezuela, Colombia, Ecuador y el norte de Perú. Es una fuente confiable y constante de agua de alta calidad y, como tal, el principal proveedor de agua para las tierras altas andinas y parte de las planicies costeras (Buytaert, et. al., 2009; Balthazar et. al., 2015). Sin embargo el crecimiento de la población y la intensificación del uso de la tierra en los Andes tropicales han causado alteraciones en el ciclo natural del agua (Buytaert, et. al., 2009; Mosquera et al., 2015). Este cambio de uso de la tierra impacta la hidrología de la cuenca, actividades como el pastoreo extensivo, cultivos, reforestación con especies exóticas; alteran las características del balance hídrico en comparación a una cuenca no intervenida o poco intervenida (Crespo P et al, 2011).

De ahí que es fundamental realizar estudios de régimen hídrico, morfología general e inventario hídrico, entendidos como las herramientas para determinar las características de las fuentes, su extensión y calidad del agua para su utilización y control; pero a su vez, la disponibilidad en cantidad y calidad en determinado lugar y en un período de tiempo para satisfacer las demandas identificables (consumo multifinalitario, humano, agrícola, energético, etc.) (Sellers, et. al., 2015; Celleri y Feyen, 2009). Es así que los modelos hidrológicos son representaciones simplificadas de los sistemas hidrológicos reales permiten estudiar el funcionamiento de las cuencas hidrográficas y su respuesta a diversos factores, y así obtener una mejor comprensión de los procesos hidrológicos. Estos permiten además predecir la respuesta hidrológica a varias prácticas de manejo de cuencas hidrográficas y tener una mejor comprensión de los impactos de estas prácticas (Arabi et al. 2008; Cho et. al. 2010; Sing et. al 2011; Fonseca et.al. 2014, Guillot et.al., 2017).

El modelado hidrológico de eventos revela cómo una cuenca responde a un evento de lluvia individual (cantidad de escorrentía superficial, pico, momento del pico, detención). En la actualidad, existen enfoques conceptuales y de modelado físico bien establecidos que se han empleado para simular procesos hidrológicos en diferentes cuencas hidrográficas como lo describen (Schuman et al. 2000; Jang et al. 2007 , Santhi et al. 2008). Por otro lado, los métodos hidrológicos e hidráulicos estiman caudales generados en una cuenca o corriente, cálculo de las velocidades y calados para un determinado tramo fluvial. Los métodos hidrológicos pueden partir de los datos de caudales con análisis estadístico de los valores máximos; o datos de precipitación, mediante modelos hidrometeorológicos de transformación lluvia-escorrentía basados en fórmulas y métodos como el racional (García, 2013).

El centro de Ingeniería Hidrológica, del Cuerpo de Ingenieros del Ejército de los EEUU, diseñó el programa de computación Sistema de Modelamiento Hidrológico (HEC-HMS), este provee una variedad de opciones para simular procesos de precipitación - escurrimiento y también tránsito de caudales entre otros (Urrutia, 2009; Prado, 2015). El programa realiza la simulación del hidrograma de una cuenca facilitándole datos físicos de la misma y con ello estima los hidrogramas de salida en una cuenca o varias subcuencas (caudales máximos y tiempos al pico) partiendo de condiciones extremas de tormentas. También calcula, por los métodos de cálculo de hietogramas de diseño, las pérdidas por infiltración, el flujo base y la conversión en escorrentía directa. Las fases de trabajo que realiza el programa son las siguientes: A) Separación de la lluvia neta (calcular qué parte de la precipitación caída va a generar escorrentía directa). B) Calcular la escorrentía directa producida por esa precipitación neta. C) Sumar a la escorrentía directa la escorrentía básica, si existía previamente. D) Calcular cómo evoluciona un hidrograma a medida que discurre a lo largo de un cauce o a través de un depósito o embalse (tránsito de hidrogramas). Al final 
suma todos los caudales generados y transitados a los largo del recorrido y nos proporciona (en tabla y en gráfico) el hidrograma en la salida de la cuenca (Aparicio et. al., 2015).

El presente estudio tuvo como objetivo simular el comportamiento hidrológico de la microcuenca Chaquilcay ubicado en la reserva de Bosques Protectores y Vegetación Aguarongo (BVPA) en los Andes ecuatorianos; para la simulación hidrológica se utilizó el software HEC - HMS (versión 4.1), así como la aplicación del programa ArcMap 10.1 para determinar las características morfológicas de la cuenca (alta y baja), se requirió del levantamiento de cartografía base como: suelo, cobertura vegetal, modelo de elevación digital y pendiente. Además información referencial de caudal mediante métodos directos e indirectos para construir la curva de gasto.

Para definir el modelo de la cuenca y la generación de sus caudales respectivos, se siguieron 3 procesos; 1) la caracterización de las abstracciones iniciales donde se utilizó el método del SCS, obtención del número de curva para la cuenca alta y baja. 2) modelo de transformación de la precipitación neta en caudal representada por el lag time, 3) tránsito de hidrogramas por medio del método de Muskingum Cunge. La construcción de los hietogramas de diseño en el modelo meteorológico se realizó a periodos de retorno de 25, 50 y 100 años a través del método de curvas IDF en función de la ecuación de intensidad zonificada para la estación Gualaceo. El propósito del estudio es establecer una metodología aplicable en microcuencas hidrográficas de la zona del austro ecuatoriano, donde no cuentan con información base hidrometeorológica y tienen una importante función ecosistémica. El agua se considera el bien ambiental más importante de este ecosistema, al representar una fuente significativa del suministro de agua para el desarrollo en la región. (Minga et al., 2002; PDYOT Jadán, 2015, Cajamarca, 2017, Loor, 2017).

\section{METODOOGÍA}

La metodología presentada pretende ser un orientativo de cómo se desarrollaron tanto la fase de manejo de datos con SIG así como la aplicación del modelo HEC-HMS.

\section{Área de estudio.}

La investigación se desarrolló en la microcuenca Chaquilcay, (sistema natural de mayor interés local) en el año 2017 con un periodo de ejecución de 6 meses, forma parte del Área de Bosque y Vegetación Protectora Aguarongo (BVPA), declarada por el Ministerio del Ambiente de Ecuador como área de protección ambiental al ser considerada como fuente principal de suministro de agua de la comunidad de Gualaceo, Sigsig y Cuenca (Minga et al., 2002). El ecosistema cuenta con 191 cauces que conforman la Cuenca del río Paute. El área de estudio se localiza en las coordenadas geográficas 742876 W - 9683894 S y 738733 W - 9674451 S (UTM WGS84-Zona 17S). Tiene un área de drenaje de $20,92 \mathrm{~km}^{2}$, que se encuentra en la parroquia Jadán, cantón Gualaceo (figura 1).

La caracterización física de la microcuenca se representa por una pendiente media del cauce principal del $8 \%$, longitud del cauce de $11,64 \mathrm{Km}$ y una extensión de la red hídrica equivalente a 54,91 Km. Referente a la topografía el área de estudio está representada por un pendiente media inclinada de $18,14 \%$ con una cota máxima y mínima de 3242 y 2274 m.s.n.m. La edafología del área presenta 4 órdenes como son: entisol, alfisol, vertisol e inceptisol siendo ésta última la predominante catalogada como un suelo de tipo C. La cobertura vegetal eminente, se clasifica en el siguiente orden por el porcentaje de área que comprende, bosques nativos $(58,67 \%)$, pasto con riego $(19.81 \%)$, pasto sin riego $(12,19 \%)$, cultivos $(4,09 \%)$, suelo descubierto $(2,37 \%)$, vegetación arbustiva y herbácea $(1,5 \%)$, vías $(0,69 \%)$ y construcciones $(0,36 \%)$.

\section{Delimitación de la Cuenca en SIG}

El modelo físico de la cuenca utiliza como entrada un modelo digital de elevaciones (MDE), el software SIG ArcMap 10.1 y la extensión especializada (Spatial Analyst) que permiten la delineación de las subcuencas y los patrones de la red de drenaje de la cuenca (Ceconi et al., 2018). La microcuenca se clasificó en dos partes utilizando el criterio planteado por Salazar (2016) que considera una diferencia altitudinal entre 0-1000 msnm, para definir la denominación: cuenca alta y baja.

\section{Configuración del modelo}

El modelo se estructuró en seis elementos hidrológicos: 1) subasin para Chaquilcay alto, 2) reservorio como una obra hidráulica para almacenamiento de agua, 3) reach (AB) que conecta entre la cuenca alta y el dique, para observar la evolución del hidrograma desde la cuenca alta al desagüe 4) reach (BC) conecta el dique con el punto de desagüe, 5) subasin para la cuenca baja conectada directamente al desagüe y 6) el desagüe representa punto donde confluye los aportes hídricos de toda la cuenca (figura 2). 


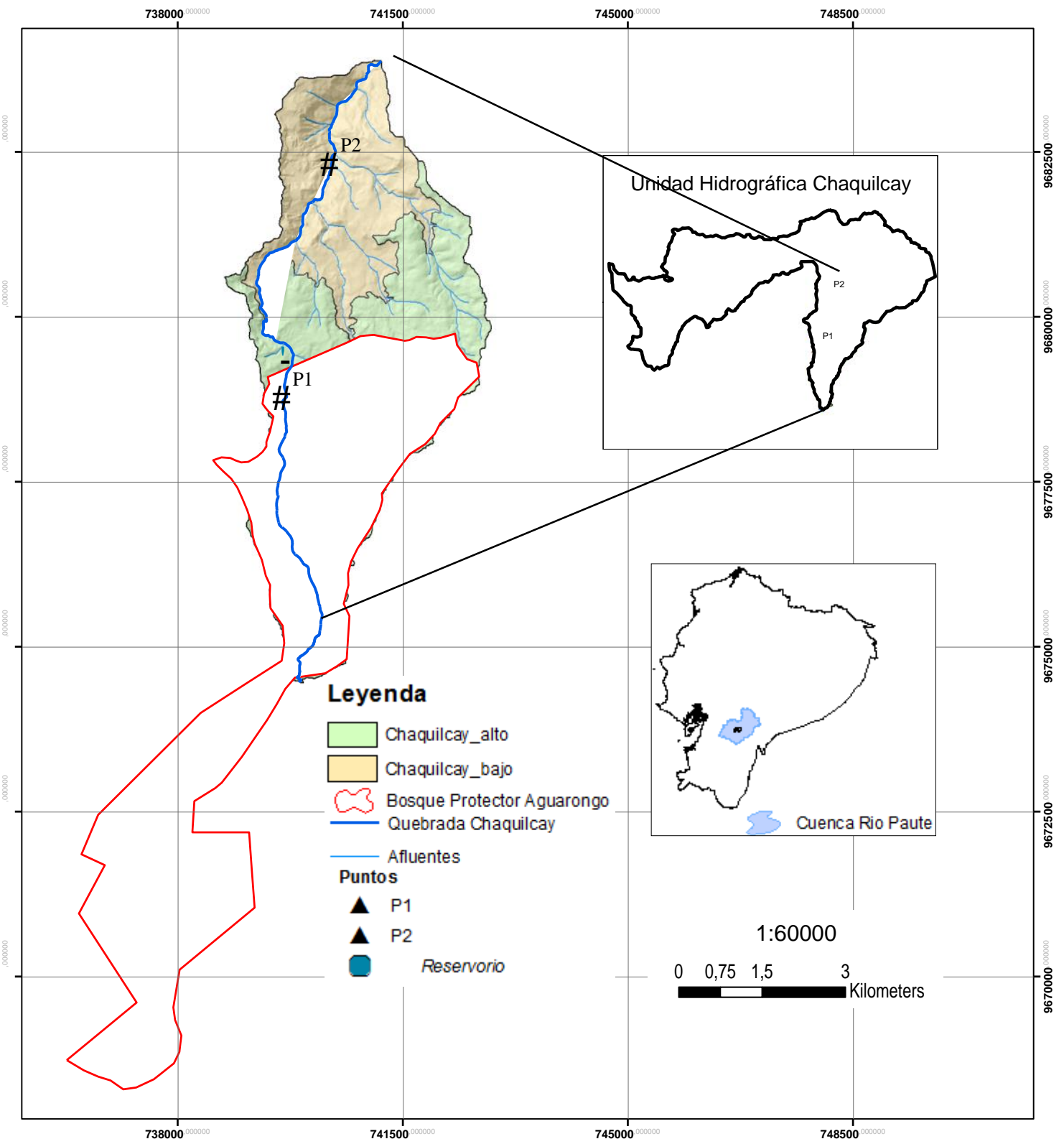

Fig. 1: Ubicación de la microcuenca Chaquilcay

El software HEC-HMS emplea los siguientes modelos (tabla 1) cada uno con el método que mejor se adapta en función de la información disponible para transformar la lluvia en escurrimiento (Sánchez 2015):

Tabla 1: Métodos y parámetros aplicados por subcuenca. Fuente: (Rodríguez \& Marrero de León, 2015)

\begin{tabular}{|c|c|c|}
\hline $\begin{array}{c}\text { Componentes del proceso de } \\
\text { escurrimiento }\end{array}$ & Método & Parámetros \\
\hline Modelo de pérdidas & Número de curva del SCS & $\begin{array}{l}\text { Numero de curva, CN } \\
\text { Abstracción inicial, la }\end{array}$ \\
\hline $\begin{array}{l}\text { Transformación de la lluvia en } \\
\text { escurrimiento }\end{array}$ & Hidrograma unitario del SCS & Tiempo de retardo, lag time \\
\hline Tránsito de avenida & Muskingum Cunge & $\begin{array}{l}\text { Longitud, D (m) } \\
\text { Ancho, W }(\mathrm{m}) \\
\text { Pendiente, }(\mathrm{m} / \mathrm{m}) \\
\text { Coeficiente de manning, n Forma del } \\
\text { cauce. }\end{array}$ \\
\hline
\end{tabular}




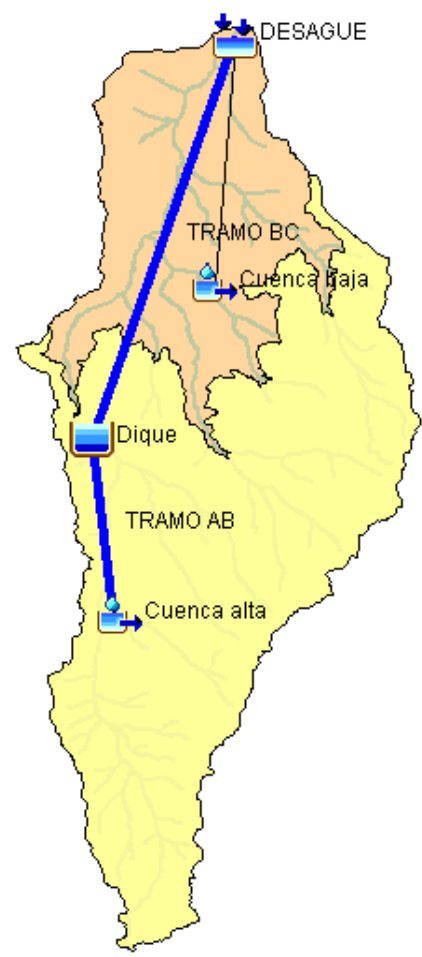

Fig. 2: Topología de la microcuenca Chaquilcay utilizada como entrada para el HEC-HMS

La entrada al modelo es la precipitación evaluada para cada subcuenca, que en este caso se la obtuvo mediante el método de los Polígonos de Thiessen, a partir de los registros de precipitación de las estaciones pluviométricas. Por lo tanto, los valores de precipitación del hietograma en cada subcuenca, $P_{s b}$, y para cada intervalo de tiempo, $i$, que se suponen uniformes en toda la superficie de cada subcuenca, se determinan como una media ponderada de las precipitaciones, $P_{e m}$, de las distintas estaciones meteorológicas, j; definida por:

$$
P_{s b}(i)=\frac{\sum_{j=1}^{n} P_{e m}(i, j) w(j)}{\sum_{j=1}^{n} w(j)}
$$

En ésta ecuación, $P_{s b}$ (i) es la precipitación media uniforme sobre una subcuenca en el intervalo i; $P_{e m}$ (i,j) es la precipitación registrada en el intervalo i y estación j; w(j) es el área de influencia de la estación j; y n es el número de estaciones meteorológicas.

Dentro del HEC - HMS como primer paso se extrae la escorrentía directa y; la precipitación que no genera escorrentía se incluye dentro las pérdidas con el modelo en este caso propuesto por el Servicio de Conservación de Suelos SCS, o también llamado; número de curva $\mathrm{CN}$, debido a que se cuenta con información del uso y tipo de suelo digitalizada. El método del SCS fue propuesto por el Departamento de Agricultura de los EE.UU., USDA, para estimar las pérdidas (o abstracciones) en un evento de lluvia o aguacero. El número de curva varía en el rango de 0 a 100 y depende de factores que influyen en la generación de escorrentía en la cuenca: tipo hidrológico del suelo (Grupo hidrológico-Capacidad de drenaje); uso y manejo del terreno; condición superficial del suelo; y condición de humedad antecedente. Por lo tanto la obtención del número de curva $(\mathrm{CN})$ se basó en la información cartográfica de pendiente, tipo de suelo y cobertura vegetal. Los valores de $\mathrm{CN}$ corresponden a una condición de humedad normal, como se muestran en la Tabla 4.

Con todo ello; la metodología propone que la escorrentía superficial directa comienza después de que se alcance un cierto valor de lluvia acumulada $l_{a}=0,2$ * $S$ la cual ha sido obtenida por medio de experimentación.

$$
\mathrm{S}=254\left(\frac{100}{\mathrm{CN}}-1\right)
$$

Siendo S la retención máxima potencia y CN el Número de curva. 
Para la generación del hidrograma en el punto de drenaje, HEC - HMS proporciona varios métodos para el cálculo, pero por experimentación se recomienda el uso de la técnica del Hidrograma Unitario $(H U)$, para cuencas pequeñas es decir menores a $20 \mathrm{~km}^{2}$, las variables utilizadas en la formula han sido adimensionalizadas.

$$
Q_{p}=\left(\frac{2.08^{*} A}{T_{p}}\right)
$$

Así, $Q_{p}$ es el caudal punta en $\mathrm{m}^{3} / \mathrm{s}$, A es la superficie de la cuenca en $\mathrm{km}^{2}$, y Tp es el tiempo máximo en horas

$$
T_{p}=\left(\frac{T_{y}}{2}+T_{f}\right)
$$

Donde, Ty es la duración del pulso de lluvia y Tf el tiempo de desfase de la cuenca.

$$
\mathrm{T}_{\mathrm{f}}=\frac{\mathrm{L}^{0.8}(2.540-22.86 \mathrm{CN})^{0.7}}{14.104 * \mathrm{CN}^{0.7 * I^{0.5}}}
$$

Siendo $L$ la longitud del cauce principal $(\mathrm{m})$, I la pendiente media de la cuenca $(\mathrm{m} / \mathrm{m})$ y $C N$ el número de curva.

Y para el cálculo de hidrogramas se utilizó el método de Muskingum. Dicha técnica hidrológica es la más utilizada para manejar variables de descargas y almacenamiento volumétrico de los hidrogramas a lo largo de cauce de los ríos mediante la combinación de dos almacenamientos conceptuales. La ecuación es la siguiente:

$$
Q_{j+1}=C_{1} l_{j+1}+C_{2} I_{j}+C_{3} Q_{j}
$$

En ésta ecuación, $l_{j}$ es el caudal entrante en un tramo en el instante j, $Q_{j}$ es el caudal aliente en el instante $j$ y $\mathrm{C}_{1}, \mathrm{C}_{2}$ y $\mathrm{C}_{3}$ son constantes que dependen del tránsito del hidrograma, valor adimensional.

\section{Modelo meteorológico}

Ante la deficiente información de datos de precipitación, se utilizó el método de curvas IDF construidas para la estación Gualaceo que es la más cercana al lugar de estudio, realizando una discretización horaria para un período de 24 horas, con ello se construyó el hietograma de diseño de la cuenca, mediante bloques alternos. La ecuación de intensidades correspondiente a la estación de Gualaceo (Ver Tabla 2) está codificada en la

\begin{tabular}{|c|c|c|c|c|c|}
\hline Código & Estación & Duración (min) & Ecuación & IdTR & $T R$ \\
\hline \multirow{3}{*}{ M0139 } & \multirow{3}{*}{ Gualaceo } & \multirow{3}{*}{\multicolumn{2}{|c|}{$\begin{array}{lr}5<51.88 & \text { ITR }=125,440 *\left(t^{\wedge}-0,4340\right){ }^{*} \text { IdTR } \\
51,88<1440 & \text { ITR }=892,87^{*}\left(t^{\wedge}-0.9310\right) * \text { IdTR }\end{array}$}} & 2,51 & 25 \\
\hline & & & & 2,637 & 50 \\
\hline & & & & 2,748 & 100 \\
\hline
\end{tabular}
zona 4 del estudio realizado por Estrella (2016).

Tabla 2: Ecuaciones para obtención de intensidad a distintos períodos de retorno correspondientes a la estación Gualaceo

\section{RESULTADOS Y DISCUSIÓN}

En la Tabla 3 se describen los valores calculados correspondientes a las características morfométricas del área de estudio. Campos citado por Viramontes et al. (2007) establece tres categorías para el coeficiente de compacidad o índice de Gravelius. La microcuenca pertenece a la clase III descrita como una cuenca oval alargada a rectangular alargada. Esta clasificación nos indica que se intensifica la magnitud de las avenidas en el desagüe. Saavedra (2001) establece que el presente valor de pendiente media se clasificaría, por el criterio de relieve o topografía, como un terreno de tipo accidentado medio. Hernández citado por Viramontes (2007) establece que para el valor obtenido de densidad de drenaje la microcuenca en estudio tiene una eficiente red de drenaje, parámetros importantes en el análisis de escorrentía de captación y otros procesos hidrológicos (Gumindoga et al., 2016). 
Tabla 3: Parámetros morfométricos de la quebrada Chaquilcay

\begin{tabular}{|l|c|c|}
\hline \multicolumn{1}{|c|}{ Parámetro } & Unidad & Valor \\
\hline Área & $\mathrm{km}^{2}$ & 20,92 \\
\hline Perímetro & $\mathrm{km}$ & 26,26 \\
\hline Longitud del cauce principal & $\mathrm{km}$ & 11,64 \\
\hline Orden del cauce principal & Adimensional & 4 \\
\hline Longitud de la red hídrica & $\mathrm{km}$ & 54,91 \\
\hline Densidad de drenaje & $\mathrm{km} / \mathrm{km}^{2}$ & 2,62 \\
\hline Cota mayor & $\mathrm{msnm}$ & 3242,00 \\
\hline Cota menor & $\mathrm{msnm}$ & 2274 \\
\hline Pendiente & $\%$ & 18,14 \\
\hline Tc & $\mathrm{hr}$ & 1,30 \\
\hline Diferencia Altitudinal & $\mathrm{m}$ & 968 \\
\hline Índice de Compacidad o de Gravelius & Adimensional & 1,62 \\
\hline Ancho de la microcuenca & $\mathrm{km}$ & 1,80 \\
\hline Pendiente media del cauce principal & $\mathrm{km} / \mathrm{km}$ & 0,08 \\
\hline
\end{tabular}

\section{Método de pérdidas y tránsito de hidrogramas}

Para la cuenca alta se obtuvo una predonimancia del suelo tipo "inceptisol", según la clasificación propuesta por Porta et al.,1994, correspondiente al grupo hidrológico C. Para la cuenca baja el tipo de suelo que predomina es el Entisol, el cual, corresponde a un suelo del grupo A. Para Chaquilcay alto se obtuvo una abstracción inicial (Po) de 71.64 y Numero de Curva (CN) de 41.49, mientras que, los valores para la cuenca baja son: Po de 62.04 y CN de 45.01. El "Lag Time" esta definido por el $60 \%$ del tiempo de concentración obteniendo un valor de 42.36 min, para la cuenca alta y 12.79 para la cuenca baja. Para el tránsito de hidrogramas se aplicó el método de Muskingum Cunge el cual fue calibrado mediante la utilización del software ArcGis 10.1, además, se realizó un levantamiento topográfico del cauce para determinar la forma del mismo, parámetros requeridos por el programa, información que se presenta en la tabla 4.

Tabla 4: Parámetros de entrada para el modelo de cuenca en HEC - HMS

\begin{tabular}{|c|c|c|c|c|c|}
\hline Elementos & \multicolumn{2}{|c|}{ Perdidas } & Transformación SCS & \multicolumn{2}{|c|}{ Muskingum Cunge } \\
\hline \multirow{3}{*}{ Cuenca Alta } & Abstracción inicial & 71,64 & \multirow{3}{*}{ Lag time 42,36 (min) } & & \\
\hline & $\mathrm{CN}$ & 41,49 & & & \\
\hline & \% Impermeabilidad & 0,43 & & & \\
\hline \multirow{5}{*}{ Tramo AB } & & & & Longitud (m) & 810 \\
\hline & & & & Pendiente $(\mathrm{m} / \mathrm{m})$ & 0,012 \\
\hline & & & & Manning & 0,08 \\
\hline & & & & Forma & Rectangular \\
\hline & & & & Ancho $(m)$ & 4 \\
\hline \multicolumn{6}{|c|}{ Caudal de referencia $\left(\mathrm{m}^{3} / \mathrm{s}\right)$} \\
\hline \multicolumn{6}{|l|}{ Dique } \\
\hline \multirow{5}{*}{ Tramo BC } & & & & Longitud (m) & 4770 \\
\hline & & & & Pendiente $(\mathrm{m} / \mathrm{m})$ & 0,08 \\
\hline & & & & Manning & 0,05 \\
\hline & & & & Forma & Rectangular \\
\hline & & & & Ancho $(m)$ & 2,87 \\
\hline \multicolumn{6}{|c|}{ Caudal de referencia $\left(\mathrm{m}^{3} / \mathrm{s}\right)$} \\
\hline \multirow{3}{*}{ Cuenca Baja } & Abstracción inicial & 62,04 & \multirow{3}{*}{ Lag time 12,79 (min) } & & \\
\hline & $\mathrm{CN}$ & 45,02 & & & \\
\hline & \% Impermeabilidad & 2,43 & & & \\
\hline
\end{tabular}




\section{Modelo meteorológico}

La construcción de las curvas IDF se realizó para periodos de retorno de 25, 50 y 100 años según la ecuación de la tabla 2 de la metodología; referente a la intensidad zonificada para la estación Gualaceo siendo esta la más próxima al área de estudio. Se considera un tiempo de concentración igual al tiempo de duración de la tormenta de 90 minutos con discretización cada 5 minutos. La intensidad observada a los 90 minutos de precipitación en las curvas IDF es: para 25 años de 33,97 mm/h, 35,69 para 50 años y 37,19 para los 100 años, (figura 3) cuya información será útil para la construcción del Hidrograma Sintético del SCS en condiciones específicas (Aguiar Wagner de et al., 2018).

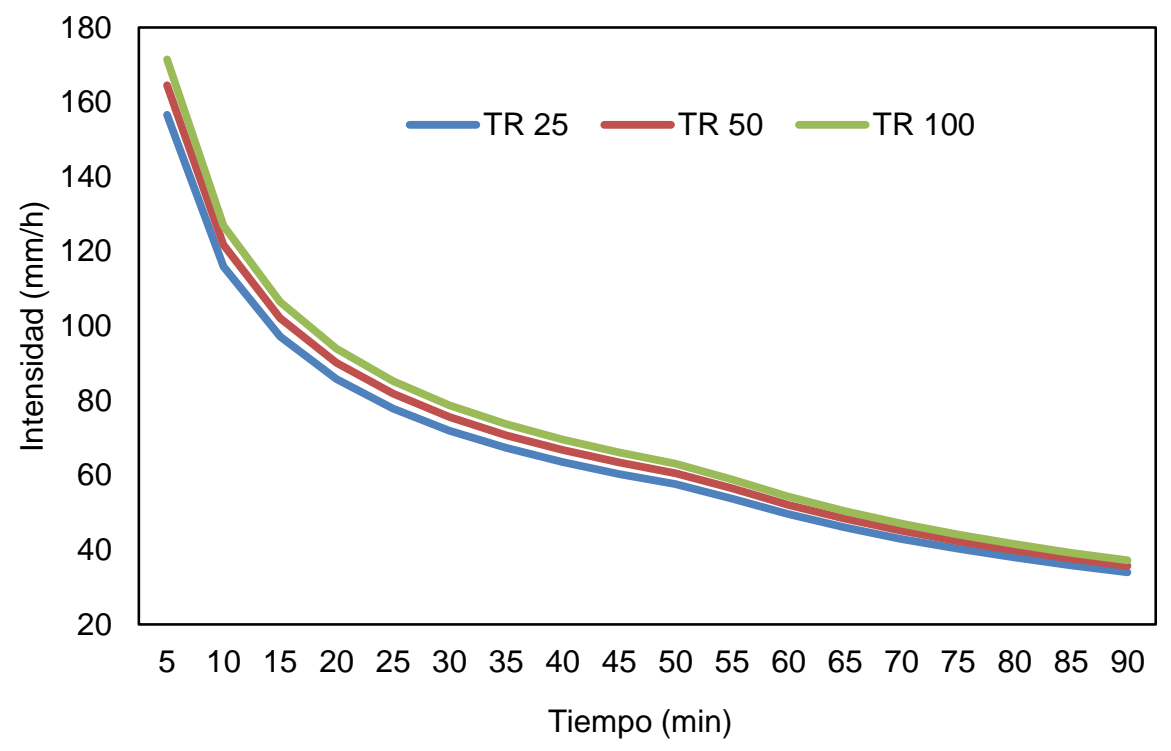

Fig. 3: Curvas IDF calculas para distintos periodos de retorno para la estación Gualaceo

\section{Calculo del caudal}

En la tabla 5 se presenta los valores simulados para distintos periódos de retorno y una condicion de humedad previa normal. Los valores de volumen de perdida para la cuenca alta aumentan conforme el período de retorno se incrementa, lo cual, está relacionado con el valor de Número de Curva (CN). En la cuenca baja la tendecia de aumento de los volumenes de pérdida se mantine conforme aumenta el período de retorno pero con la diferencia que los valores simulados son menores.

Tabla 5: Parámetros de salida en la simulación del modelo HEC-HMS. * Caudal expresado en $\mathrm{m}^{3} / \mathrm{s}$, ** Volumen expresado en $\mathrm{m}^{3}$

\begin{tabular}{|l|l|r|r|r|}
\hline \multirow{2}{*}{$\begin{array}{c}\text { Elementos de la } \\
\text { cuenca }\end{array}$} & \multicolumn{1}{|c|}{ Parámetro } & \multicolumn{3}{c|}{ Condición Normal II } \\
\cline { 2 - 5 } & & 25 años & 50 años & 100 años \\
\hline \multirow{5}{*}{ Cuenca alta } & * Caudal pico de descarga & 0,5 & 0,5 & 0,6 \\
\cline { 2 - 5 } & ** Volumen precipitación & 883,1 & 927,7 & 966,8 \\
\cline { 2 - 5 } & Volumen de pérdida & 879,3 & 923,7 & 962,6 \\
\cline { 2 - 5 } & Volumen descarga & 3,8 & 4 & 4,1 \\
\hline \multirow{5}{*}{ Cuenca baja } & Caudal pico de descarga & 1,7 & 1,7 & 1,8 \\
\cline { 2 - 5 } & Volumen precipitación & 407,9 & 428,5 & 446,6 \\
\cline { 2 - 5 } & Volumen de pérdida & 398 & 418 & 435,1 \\
\cline { 2 - 5 } & Volumen descarga & 9,9 & 10,5 & 11,4 \\
\hline \multirow{3}{*}{ Desagüe } & Caudal pico & 1,7 & 1,8 & 1,9 \\
\cline { 2 - 5 } & Volumen & 13,7 & 14,5 & 15,6 \\
\hline
\end{tabular}

En la figura 4 se muestra los valores de caudales de crecida simulados en el desagüe de la cuenca en el cual se visualiza un desfase en amplitud del hidrograma conforme el período de retorno aumenta, se realizaron calibraciones semiautomáticas, a través del módulo "optimization trial" del HEC-HMS, como lo indica Feldman (2000). Se observa dos puntas de caudal en el hidrograma correspondiente a un período 
de retorno de 25 años a las 12 horas y a las 18 horas. Para un período de retorno de 50 años se observan dos puntas de caudal a las 12 horas y 17 horas de precipitación, mientras que, para un período de retorno de 25 años existe una sola punta de caudal a las 12 horas de precipitación.

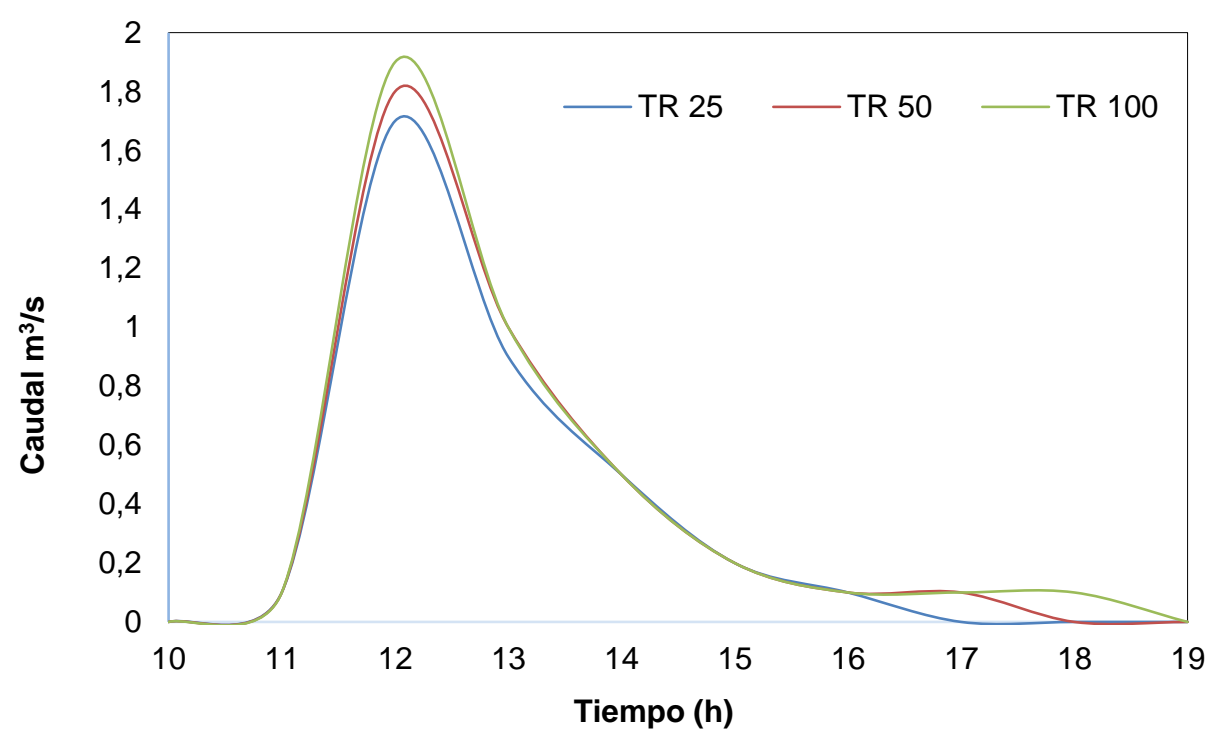

Fig. 4: Hidrograma de salida de la cuenca para distintos períodos de retorno.

\section{Análisis de sensibilidad}

Para mejorar los valores simulados se modificaron los valores de los parámetros como el número de curva y el tiempo de retraso (lag time) del modelo como lo indica Sarría y Palazón (2008). Se obtuvo valores de caudal y volumen de escorrentía presentados en la tabla 6.

Tabla 6: Valores de caudal punta para los distintos escenarios del análisis de sensibilidad

\begin{tabular}{|c|c|c|c|c|c|c|c|c|c|c|}
\hline \multirow{2}{*}{$\begin{array}{c}T R \\
\text { (años })\end{array}$} & \multicolumn{2}{|c|}{$C N$ II } & \multicolumn{2}{c|}{$C N+20 \%$} & \multicolumn{2}{c|}{$C N-20 \%$} & \multicolumn{2}{c|}{ Lag Time $+20 \%$} & \multicolumn{2}{c|}{ Lag Time -20\% } \\
\cline { 2 - 11 } & $Q\left(\mathrm{~m}^{3} / \mathrm{s}\right)$ & $V\left(\mathrm{~m}^{3}\right)$ & $Q\left(\mathrm{~m}^{3} / \mathrm{s}\right)$ & $V\left(\mathrm{~m}^{3}\right)$ & $Q\left(\mathrm{~m}^{3} / \mathrm{s}\right)$ & $V\left(\mathrm{~m}^{3}\right)$ & $Q\left(\mathrm{~m}^{3} / \mathrm{s}\right)$ & $V\left(\mathrm{~m}^{3}\right)$ & $Q\left(\mathrm{~m}^{3} / \mathrm{s}\right)$ & $V\left(\mathrm{~m}^{3}\right)$ \\
\hline 25 & 1,7 & 13,7 & 1,7 & 13,7 & 1,7 & 13,7 & 1,7 & 13,7 & 1,7 & 13,7 \\
\hline 50 & 1,8 & 14,5 & 1,8 & 14,6 & 1,8 & 14,5 & 1,8 & 14,5 & 1,8 & 14,5 \\
\hline 100 & 1,9 & 15,6 & 1,9 & 15,8 & 1,8 & 15,4 & 1,9 & 15,6 & 1,9 & 15,6 \\
\hline
\end{tabular}

En las gráficas de los hidrogramas para los periodos de retorno respectivos presentadas en la figura 5 se observa un comportamiento similar de los valores de caudal con un $+/-20 \%$ de $\mathrm{CN}$ siendo el parámetro más sensible el volumen de escorrentía generado, mientras que, la simulación con el tiempo de retardo (lag time) en el rango $+/-20 \%$ afecta en amplitud a los hidrogramas.

\section{CONCLUSIONES}

En base a los resultados expuestos se puede obtener las siguientes conclusiones: La metodología aplicada responde de forma adecuada al objetivo, el método del Número de Curva del SCS es aplicable para cuencas menores a $250 \mathrm{~km}^{2}$ y con limitada información como este caso de análisis, dando origen a resultados aceptables de caudales punta. Se obtuvo el caudal de diseño como variable hidrológica para los distintos periodos de retorno generado con la aplicación del software HEC-HMS 4.1. Del análisis de sensibilidad se determinaron como parámetros sensibles el número de curva y lag time. Los resultados establecen que del total de precipitación caída en la cuenca el $25 \%$ genera escorrentía directa y el $75 \%$ se atribuye a abstracciones condición dada por la presencia de masas forestales correspondiente al $60,17 \%$ de bosque nativo y vegetación arbustiva así como por procesos de intercepción, almacenaje de superficie, evaporación, evapotranspiración y sistemas de captaciones de agua. 

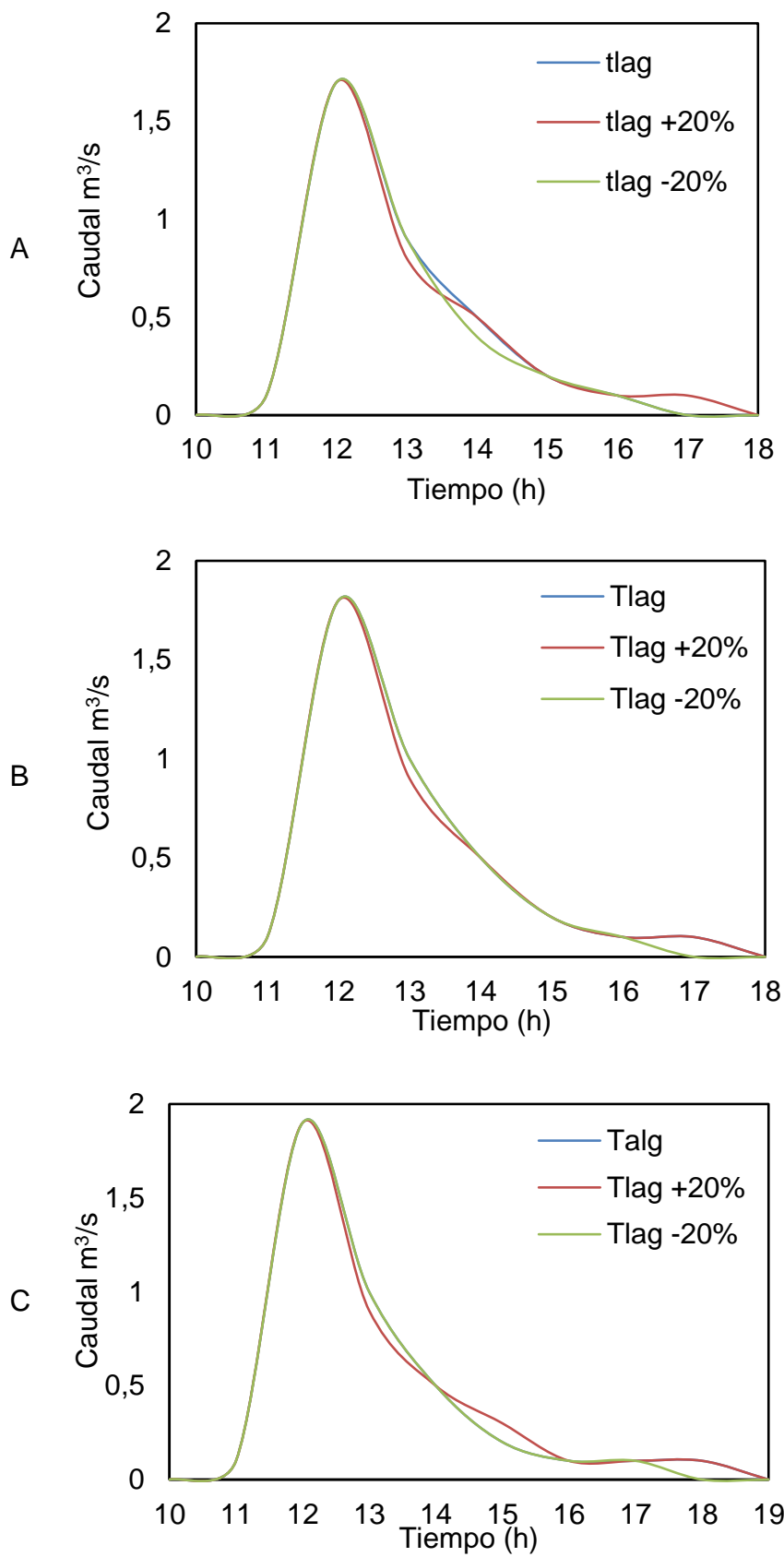

Fig. 5: Hidrogramas simulados con un análisis de sensibilidad para los distintos periodos de retorno. A) TR: 25 años, B) TR: 50 años y C) TR: 100 años.

\section{REFERENCIAS}

Aber J, RP. Neilson y otros cuatro autores, Forest Processes and Global Environmental Change: Predicting the Effects of Individual and Multiple Stressors, doi: 10.1641/0006-3568(2001)051[0735:FPAGEC]2.0.CO;2, BioScience, 51 (9), 735751 (2001)

Acosta R, H. Hampel y otros cuatro autores, Protocolo de evaluación de la calidad biológica de los ríos de la región austral del Ecuador. ETAPA EP. SENAGUA. - DHS. Universidad de Cuenca; 62 p (2014)

Aguiar, Wagner de, S.C. Sampaio, J.C. Paisani y R.R. Reis dos, Implications for peak flows of the marrecas river basin due to changes in the brazilian forest code. Engenharia Agrícola, https://dx.doi.org/10.1590/1809-4430eng.agric.v38n2p277-283/2018, 38 (2) 277-283 (2018).

Aparicio F, Fundamentos de la hidrología de superficie. México D.F., ME: Editorial Limusa; 304 p (2015)

Arabi, M., J.R. Frankenberger, B. A. Engel y J.G. Arnold, Representation of agricultural conservation practices with SWAT. Hydrological Processes: An International Journal, doi: 10.1002/hyp.689022(16), 3042-3055 (2008).

Balthazar, V., V. Vanacker, A. Molina y E.F. Lambin, Impacts of forest cover change on ecosystem services in high Andean mountains. Ecological indicators, doi: 10.1016/j.ecolind.2014.07.04348, 63-75 (2015) 
Buytaert W, R. Célleri, L. Timbe, Predicting climate change impacts on water resources in the tropical Andes: Effects of GCM uncertainty. Geophysical Research Letters, 36, L07406, doi: 10.1029/2008GL037048 (2009)

Cajamarca R. E. Estudio del balance hídrico superficial de las cuencas hidrográficas, sector Jadán y Zhidmad en el área de interceptación con el Bosque y Vegetación Protectora Aguarongo (BVPA) .BS tesis (2017)

Ceconi D.E., D.G. Allasia, F. Bernardi, P. Fensterseifer, Analysis of vulnerability for environmental planning of a water supply basin, Ambient. Soc., http://dx.doi.org/10.1590/1809-4422asoc0078r2vu18l1ao, 21: e00782 (2018)

Celleri R, P. Willems, W. Buytaert, J. Feyen, Space-time rainfall variability in the Paute basin, Ecuadorian Andes. Hydrological Processes, doi: 10.1002/hyp.6575, 21: 3316-3327 (2007)

Celleri R, J. Feyen, The Hydrology of Tropical Andean Ecosystems: Importance, Knowledge Status, and Perspectives. Mountain Research and Development, doi: 10.1659/mrd.00007, 29(4): 350-355 (2009)

Cargua, F.E., M.V. Rodriguez, C.G. Recalde y L.M. Vinueza, Cuantificación del Contenido de Carbono en una Plantación de Pino Insigne (Pinus radiata) y en Estrato de Páramo de Ozogoche Bajo, Parque Nacional Sangay, Ecuador. Inf. Tecnol., 25 (3) 83-92, ISSN 0718-0764, http://dx.doi.org/10.4067/S0718-07642014000300011 (2014)

Cho, J., S. Mostaghimi, y M.S. Kang, Development and application of a modeling approach for surface water and groundwater interaction. Agricultural water management, doi:10.1016/j.agwat.2009.08.018, 97(1), 123-130 (2010)

Crespo, P. J., J. Feyen y otros seis autores, Identifying controls of the rainfall-runoff response of small catchments in the tropical Andes (Ecuador). Journal of Hydrology, doi: 10.1016/j.jhydrol.2011.07.021, 407(1-4), 164-174 (2011)

Dirnböck, T, S.Y. Dullinger, G.A. Grabherr, regional impact assessment of climate and land-use change on alpine vegetation. Journal of Biogeography, doi:10.1046/j.1365-2699.2003.00839.x, 30, 401-417 (2003)

Duque, P.J., R. Cajamarca, B.C. Wemple y M. Delgado, Estimation of the water balance for a small tropical Andean catchment. La Granja: Revista de Ciencias de la Vida, doi.org/10.17163/lgr. n29.2019.05. Vol. 29(1), 56-69 (2019)

Estrella, D., Determinación de curvas de frecuencia y zonificación de intensidades en la cuenca media alta del río Paute, Cuenca-Ecuador, (2016)

Fonseca, A., C. Botelho, R.A. Boaventura, y V.J. Vilar, Integrated hydrological and water quality model for river management: a case study on Lena River. Science of the Total Environment, doi: 10.1016/j.scitotenv.2014.03.111, 485, 474-489 (2014)

Guillot, J.D, C.A. Robles y J.D. Callejas, Adquisición de Señales Ambientales para un Sistema de Alerta Temprana. Inf. Tecnol, 28 (5), 45-54. ISSN 0718-0764, doi.org/10.4067/S0718-07642017000500007 (2017)

Gumindoga, W, H. Makurira, M. Phiri, y I. Nhapi, Estimating runoff from ungauged catchments for reservoir water balance in the Lower Middle Zambezi Basin. Water SA, https://dx.doi.org/10.4314/wsa.v42i4.15, 42(4) 641-649 (2016)

Jang S, M. Cho y otros seis autores, Using SWMM as a tool for hydrologic impact assessment. Desalination, doi: 10.1016/j.desal.2007.05.005, 212:344-356 (2007)

Kulonen A, R.A. Imboden y otros tres autores, Enough space in a warmer world? Microhabitat diversity and small-scale distribution of alpine plants on mountain summits, Diversity and Distributions, doi: 10.1111/ddi.12673, 24(2), 252-261 (2017)

Loor, Y.S., Estudio del balance hídrico superficial de las cuencas hidrográficas sector San Juan y San Bartolomé en el área de interceptación con el Bosque y Vegetación Protector Aguarongo. BS tesis. (2017)

López X.E., D.M. Patiño, Aplicación de modelos hidrológicos de las microcuencas del Área de Bosque y Vegetación Protector Aguarongo con enfoque al cambio climático. BS tesis. (2017)

Minga N, X. Sánchez y otros tres autores, Plan de Manejo del Bosque Protector Aguarongo y su área de influencia. Cuenca; 119 p. (2002)

Mosquera G.M., P.X. Lazo y otros tres autores, Runoff from tropical alpine grasslands increases with areal extent of wetlands. CATENA, doi: 10.1016/j.catena.2014.10.010, 125, 120-128 (2015)

OMM. Guía de Prácticas Climatológicas. (Guía N.- 100) Ginebra, SUI: Organización Meteorológica Mundial (OMM); 128 p. $(2011)$

PDYOT Jadán. Plan de Desarrollo y Ordenamiento Territorial. Parroquia Jadán. Gualaceo; 271 p. Disponible en: http://app.sni.gob.ec/sni-link/sni/PORTAL_SNI/data_sigad_plus/sigadplusresolucion/

0160037430001_DIAGNOSTICO_Jadan\%2030\%200ct_30-10-2015_11-40-03.pdf. (2015)

Quintero, W., C.A. Robles y A.M. Viloria, Sistema de Información para Detección de Crecientes Súbitas en la Cuenca del Río Manzanares en Santa Marta, Colombia. Inf. Tecnol. 28(6): 95-102. ISSN 0718-0764, http://dx.doi.org/10.4067/S0718-07642017000600011 (2017)

Rodríguez, Y., y N. Marrero de León, Simulación hidrológica en dos subcuencas de la cuenca del río Zaza de Cuba. Ingeniería Hidráulica y Ambiental 36(2), 109-123. (2015)

Sánchez, F., HEC-HMS Manual Elemental. Departamento de Geología, Universidad de Salamanca, España, 1-3 (2015) 
Santhi C, N. Kannan, J.G. Arnold y M.D. Luzio, Spatial calibration and temporal validation of flow for regional scale hydrologic modeling, doi: 10.1111/j.1752-1688.2008.00207.x, J Am Water Resour Assoc 44(4), 829-846 (2008)

Sarría, F. y J.A. Palazón, Modelización de sistemas ambientales, Geografía, Ecología e Hidrología (2008)

Sellers, C., E. Corbelle, S. Buján y D. Miranda, Morfología Interpretativa de Alta Resolución con Datos Lidar en la cuenca del Río Paute - Ecuador, IERSE 30 Instituto de Estudios de Régimen Seccional Del Ecuador, 131-180 (2015)

Urrutia, R., y M. Vuille, Climate change projections for the tropical Andes using a regional climate model: Temperature and precipitation simulations for the end of the 21st century, Journal of Geophysical Research, doi: 10.1029/2008JD011021, 114, D02108 (2009)

Vera C, G. Silvestri, B. Liebmann y P. Gonzalez, Climate change scenarios for seasonal precipitation in South America from IPCC-AR4 models. Geophysical Research Letters, doi: 10.1029/2006GL025759, 33, L13707 (2006) 